\title{
Classification models for clear cell renal carcinoma stage progression, based on tumor RNAseq expression trained supervised machine learning algorithms
}

\author{
Zeenia Jagga, Dinesh Gupta* \\ From Great Lakes Bioinformatics Conference 2014 \\ Cincinnati, OH, USA. 16-18 May 2014
}

\begin{abstract}
Background: Clear-cell Renal Cell Carcinoma (ccRCC) is the most- prevalent, chemotherapy resistant and lethal adult kidney cancer. There is a need for novel diagnostic and prognostic biomarkers for CCRCC, due to its heterogeneous molecular profiles and asymptomatic early stage. This study aims to develop classification models to distinguish early stage and late stage of $c C R C C$ based on gene expression profiles. We employed supervised learning algorithms- J48, Random Forest, SMO and Naïve Bayes; with enriched model learning by fast correlation based feature selection to develop classification models trained on sequencing based gene expression data of RNAsea experiments, obtained from The Cancer Genome Atlas.

Results: Different models developed in the study were evaluated on the basis of 10 fold cross validations and independent dataset testing. Random Forest based prediction model performed best amongst the models developed in the study, with a sensitivity of $89 \%$, accuracy of $77 \%$ and area under Receivers Operating Curve of 0.8 .

Conclusions: We anticipate that the prioritized subset of 62 genes and prediction models developed in this study will aid experimental oncologists to expedite understanding of the molecular mechanisms of stage progression and discovery of prognostic factors for ccRCC tumors.
\end{abstract}

\section{Background}

Renal cell carcinoma is a common adult kidney cancer, accounting for $2-3 \%$ of all new cancer cases diagnosed worldwide [1]. Detection of renal cell carcinoma at an early stage is difficult and generally diagnosed incidentally [2]. Most cases can be treated effectively only if detected timely, increasing the survival rates of patients [3]. The clear-cell Renal Cell Carcinoma (ccRCC) is the most common subtype of the renal cell carcinoma, characterized by clear cell morphology of the cytoplasm [4]. So far, early stage diagnosis is difficult due to the molecular complexity and divergent clinical behavior of ccRCC

\footnotetext{
* Correspondence: dinesh@icgeb.res.in

Bioinformatics Laboratory, Structural and Computational Biology Group, International Centre for Genetic Engineering and Biotechnology (ICGEB), Aruna Asaf Ali Marg, New Delhi, India
} stage specific distinction in ccRCC.

Advancements in high throughput technologies like Next Generation Sequencing have opened novel avenues in cancer research with renewed emphasis on diagnosis, prognosis and therapeutics. The launch of large scale comprehensive multi-node programs like International Cancer Genomic consortium [ICGC] (http://icgc.org/) and The Cancer Genome Atlas [TCGA] (http://cancergenome. nih.gov/) enables systematic studies on genomic, epigenomic and transcriptomic levels for different cancer types that hold clinical and societal importance globally [6]. These projects are making data available to researchers in different levels 1, 2, 3, 4 (i.e. raw, processed, interpreted candidate biomarkers for diagnosis and/or prognosis for 
and summarized respectively) enabling genome informed personalized cancer medicine research $[7,8]$.

Along with advances and affordability of high throughput technologies and data, progress is also being made towards personalized and predictive medicine for clinical management of cancer patients. In order to analyze diverse and multidimensional cancer related data, machine learning techniques are being extensively applied for cancer prognosis as well as diagnosis $[9,10]$.

In ccRCC, clinical tumor staging by TNM staging system is used - as prognostic factor; determines treatment regimen of patient; confirmed to guide the surveillance protocols and to assess risk of metastatic renal cell carcinoma [3,11-13]. Although tumor stage being an effective prognostic factor, to our knowledge there has been no systematic studies characterizing gene expression data for tumor stage progression. Thereby, we hypothesized that identifying gene expression signature that correlates with clinical tumor stage progression might lead to discovery of a panel of prognostic molecular signatures for ccRCC tumors.

In this study, we have developed prediction models to discriminate clinical tumor stages- early stage (I, II) and late stage (III, IV) of ccRCC. The prediction models are trained on gene expression data of RNAseq experiments from TCGA by implementing state-of-art supervised machine learning algorithms. The gene expression signatures identified by feature selection approach, which enriched classifier training, helped us to efficiently classify the tumors based on their clinical tumor stage. Further, we found that amongst the implemented machine learning techniques, predictive models based on Random Forest algorithm performed the best with accuracy of $76.84 \%$ on independent data test, area under Receiver Operating Characteristic Curve (auROC) of 0.778.

\section{Methods}

The overall methodology followed in the article is summarized in Figure 1.

\section{Data mining}

The dataset for this study was downloaded from TCGA data portal (https://tcga-data.nci.nih.gov/tcga/tcgaHome2.jsp). The Level 3 RNAseq expression dataset from cancer type KIRC i.e. Kidney Renal clear cell carcinoma on Illumina HiSeq 2000 RNA sequencing platform was obtained from TCGA data portal [14]. The RNAseq expression data was available for 475 tumor samples. Each patient gene expression profile consists of gene expression data for 20,534 genes. Only tumor samples were taken into account in the study. The clinical information for selected subjects was retrieved from the "clinical Biotab" section of the data matrix based on the BCR (Biospecimen Core Resource) IDs of the patients.
We generated a gene expression data matrix in Comma Separated Value (CSV) file format from the data retrieved from the TCGA data portal, with 20,534 genes as column labels and 475 patients BCR ids as row labels. This was achieved using in-house shell scripts. The 'Reads Per Kilobase per Million' i.e. RPKM values of the mapped reads, retrieved as an estimate for gene expression, were used as feature vectors for classifiers trainings. In order to generate a study dataset, we marked class label of "Early Stage" for patients with clinical tumor stage I \& II, and class labels of "Late Stage" for tumor stage III \& IV.

Testing and Training dataset gene expression matrix with $9756650(20,534 \times 475)$ data points was randomly stratified and split into $80 \%$ training-cum-validation dataset (Additional File 2) and 20\% independent testing dataset (Additional File 3) using a PERL script, developed in house.

\section{Machine learning}

All the Machine learning steps including data preprocessing, feature selection, generating classification models and independent testing was performed on Waikato Environment for Knowledge Analysis (WEKA) version 3.7.9 [15].

Feature selection: For reducing the dimensionality in feature space and improving instance to feature ratio for better machine learning, we combined WEKA attribute evaluator 'SymmetricalUncertAttributeSetEval' with search method of 'FCBFSearch'. The algorithm Fast Correlation Based Feature (FCBF) selection utilizes predominant correlation to identify relevant features in high dimensional datasets [16]. This feature selection method is used in conjunction with evaluator 'Symmetrical Uncert Attribute Eval', which selects subset of features based on symmetrical uncertainty with respect to another features $[16,17]$.

We then analyzed this subset gene list for Gene Ontology (GO)- biological process annotations with WebGestalt (http://bioinfo.vanderbilt.edu/webgestalt/), a Web-based Gene Set Analysis Toolkit [18]. We visualized the impact of our candidate genes at genomic (mutation, copy number alterations), transcriptomic (expression) and protein levels (expression) in ccRCC TCGA dataset through cBio Cancer Genomics Portal $[19,20]$. We also estimated overall Kaplan-Meier Survival curve based on cases with and without alteration in these genes using cBio Cancer Genomics Portal.

\section{Classification algorithms}

We employed four different state-of-the-art supervised machine learning algorithms (J48, Naïve Bayes, Sequential minimal optimization and Random Forest) for generating the classification models. J48 is the java based implementation decision tree learning algorithm C4.5 [21]. Naïve 


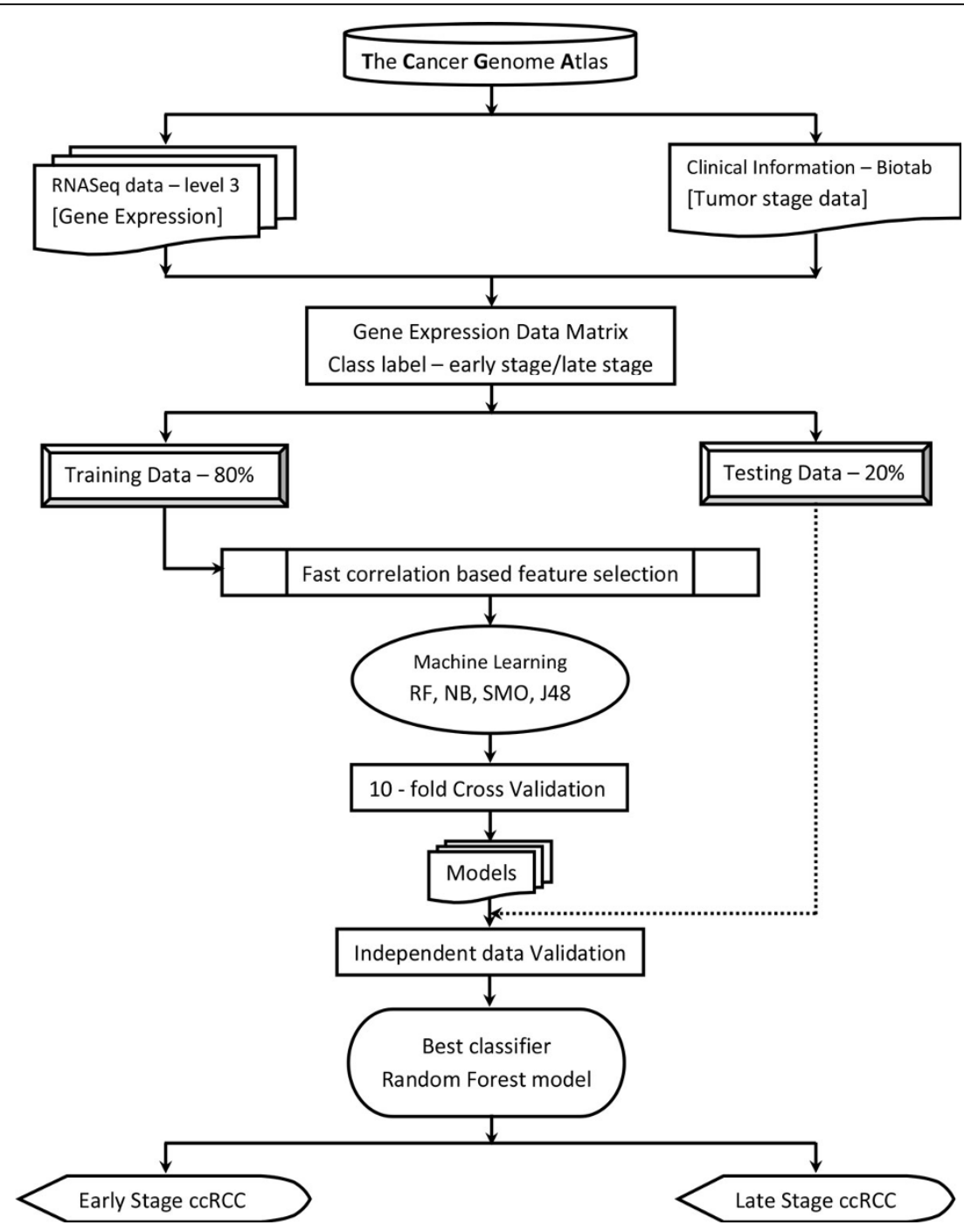

Figure 1 Schematic representation of the overall strategy used in the study. Data was downloaded from TCGA data portal. Gene expression values of the tumors constituted the descriptor values. Class labels were derived from the clinical information given in the Biotab of the TCGA data portal. Resulting data was processed to generate training/testing data files. Feature selection and generation of classification models was performed on WEKA Workbench.

Bayes algorithm works on the assumption that all the features are statistically independent and is based on Bayes theorem [22]. Sequential Minimal Optimization (SMO) algorithm is an implementation of Support Vector Classifier $[23,24]$. Random Forest is randomly constructed ensemble of independent decision trees [25].

Training-cum-validation: The four supervised machine learning algorithms (J48, LibSVM, Naïve Bayes, and Random Forest) were trained on the subset features from feature selection and further validated by 10 fold crossvalidation. The training models thus generated were compared based on the accuracy and auROC. Random Forest classifier was optimized for number of trees and the models were generated using number of trees as 1000 .
Independent dataset test: Independent data testing is recommended to exclude the "memory" effect or bias for trained classification models [26]. So, we re-evaluated the performance of best-trained and cross-validated model on independent dataset.

\section{Performance evaluation}

In order to evaluate the generated prediction models we employed various evaluation metrics recommended for evaluating the classifier's performances i.e. accuracy, sensitivity or recall, specificity, Matthews Correlation Coefficient (MCC), F-value and auROC $[27,28]$. The auROC is area under curve and is evaluated for comparing performance of several classifiers. 


\section{Results}

\section{Gene expression data matrix}

The RNAseq dataset for 475 clinically diagnosed ccRCC patients were retrieved from TCGA data portal. The dataset includes gene expression levels for 20,534 genes in tumor tissue samples of the patients. Thus, we generated a study dataset of gene expression data matrix of 20,534 $\times$ 475 , columns and rows respectively. The data characteristics of patients whose gene expression values have been used in this study are given as Additional File 1. Further, the study dataset was divided into $80 \%$ training-cumvalidation and $20 \%$ independent testing dataset. The distribution of patients across training and testing dataset by clinical tumor stage is given in Table 1.

\section{Feature selection}

Feature selection performed on training-cum-validation dataset of 219 early stage and 161 late stage instances by fast correlation based feature selection largely reduced the feature vectors space from 20,534 genes to 62 genes (see additional files: 4 for list of genes, 5 for distribution plot of the expression values for all the selected 62 genes). From the GO Slim Annotation of subset genes, we identified "multicellular organismal process" (29 genes), "response to stimulus" (28 genes), "metabolic process" (26 genes) and "biological regulation" (26 genes) as most frequent GO annotations for Biological Processes (Additional file 6). Oncoprint of the 62 identified candidate gene list by feature selection determined the alterations of genomic profiles in $83.6 \%$ of ccRCC cases (Additional file 6). Further validation of the relevance of selected gene features was achieved by performing survival Kaplan-Meir estimate. The survival estimate revealed that the median months survival in cases with alterations is 73.17 months and cases without alterations is 90.38 months (Additional File 6).

\section{Training-cum-validation}

We evaluated training models by 10 fold cross-validation for the classifiers trained on the four supervised machine learning algorithms- SMO, Random Forest, J48 and Naïve Bayes. All the models were based on standard error base classifiers. The performances of the generated prediction models were compared on the basis of accuracy and

Table 1 Summary of Training and Testing dataset statistics.

\begin{tabular}{llrr}
\hline \multicolumn{1}{c}{ Class label } & \multicolumn{1}{c}{ Clinical status } & \multicolumn{1}{c}{ Training } & \multicolumn{1}{c}{ Testing } \\
\hline Early Stage & Stage I & 180 & 45 \\
Early Stage & Stage II & 39 & 10 \\
Late Stage & Stage III & 97 & 24 \\
Late Stage & Stage IV & 64 & 16 \\
& & $\mathbf{3 8 0}$ & $\mathbf{9 5}$ \\
\hline
\end{tabular}

auROC values (Table 2). The classification accuracy of the generated prediction models ranges from $67.6 \%$ for J48, to 79.7\% for Random Forest; and auROC value ranges from 0.7 for $\mathrm{J} 48$ to 0.876 for Random Forest. Based on accuracy and auROC, we inferred that Random Forest based prediction model has outperformed other three machine learning algorithms implemented in the study.

\section{Independent data testing}

Furthermore, we evaluated our prediction models on an independent dataset with 55 early stage patients and 40 late stage patients. The performances of the prediction models were compared on the basis of standard statistical measures- accuracy, sensitivity, specificity, F-measure, and auROC (Table 3). We observed coherence in the performance of the models between independent data testing and 10 fold cross validation based on auROC values.

We evaluated sensitivity and specificity plot to determine prediction model with low error rates i.e. high sensitivity and specificity. Sensitivity of all the models was in the range of $79-94 \%$, with highest sensitivity of $93.56 \%$ for Naïve Bayes. Specificity of the models varied in a wide range with lowest of $39.0 \%$ for $J 48$ and the highest of $60.98 \%$ for Random Forest (Figure 2). Although, Naïve Bayes based model shows the highest sensitivity, the best sensitivity-specificity trade-off was observed for Random Forest Classifier with $88.89 \%$ sensitivity and $60.98 \%$ specificity.

The prediction accuracies of all the four predictive models on independent dataset were- 62.11, 72.63, 76.84, 73.68 for J48, Naive Bayes, Random Forest and SMO respectively (Table 3). F-measures of the models developed in the study is between 0.7 to 0.82 . It is notable that amongst the four evaluated prediction models, the model based on Random Forest displays highest accuracy and F-measure.

ROC plot is evaluated to characterize threshold independent performance of the prediction models. The ROC plot of the classifiers showing tradeoff between true positive rate and false positive rate in Figure 3 and auROC values in Table 3 suggests that prediction models based on Random Forest algorithm performed better than Naïve Bayes, SMO and J48. The prediction models had

Table 2 Performance of prediction models generated by 10 -fold cross validation from training-cum validation dataset.

\begin{tabular}{lcc}
\hline \multicolumn{1}{c}{ Classifier } & Accuracy & auROC $^{\#}$ \\
\hline J48 & 67.6316 & 0.700 \\
Naïve Bayes & 77.8947 & 0.843 \\
Random Forest & $\mathbf{7 9 . 7 3 6 8}$ & $\mathbf{0 . 8 7 6}$ \\
SMO & 76.0526 & 0.745 \\
\hline
\end{tabular}

\# auROC denotes area under Receivers Operating Characteristic curve. Highest numerical value in each column is highlighted as bold letters. 
Table 3 Performance of prediction models by standard statistical evaluation parameters for independent testing dataset.

\begin{tabular}{lccccc}
\hline \multicolumn{1}{c}{ Classifier } & Accuracy & Sensitivity & Specificity & F-value & auROC $^{\#}$ \\
\hline J48 & 62.11 & $79.63 \%$ & $39.02 \%$ & 0.704918 & 0.563 \\
Naïve Bayes & 72.63 & $\mathbf{9 3 . 5 6 \%}$ & $48.78 \%$ & 0.790323 & 0.749 \\
Random & $\mathbf{7 6 . 8 4}$ & $88.89 \%$ & $\mathbf{6 0 . 9 8 \%}$ & $\mathbf{0 . 8 1 3 5 5 9}$ & $\mathbf{0 . 7 7 8}$ \\
Forest & & & & & \\
SMO & 73.68 & $87.04 \%$ & $56.10 \%$ & 0.789916 & 0.716 \\
\hline
\end{tabular}

\# auROC denotes area under Receivers Operating Characteristic curve. Highest numerical value in each column is highlighted as bold letters.

an auROC value of more than 0.5 i.e. better than random predictions, assuring their robust performance.

Thus, on the basis of statistical evaluators used for characterizing the performance of the prediction models, classifiers were ranked in following order- Random Forest, Naive Bayes, SMO and J48.

Hence, Random Forest based prediction model is an efficient classifer with $88.89 \%$ sensitivity, $76.84 \%$ accuracy and auROC of 0.778 for classifying early stage and late stage of the ccRCC tumors, using gene expression profiles.

\section{Discussion}

Predictive classification models in cancer biology are employed with either of the 3 foci- predicting cancer susceptibility, recurrence or survivability [9]. Previous studies in ccRCC employs gene expression profiles to predict survival $[29,30]$ and determine newer tumor subtypes $[31,32]$. We have used gene expression profiles of ccRCC stage specific tumor tissues to train supervised machine learning algorithms, towards our goal to develop classification models for early stage to late stage tumor progression of ccRCC.

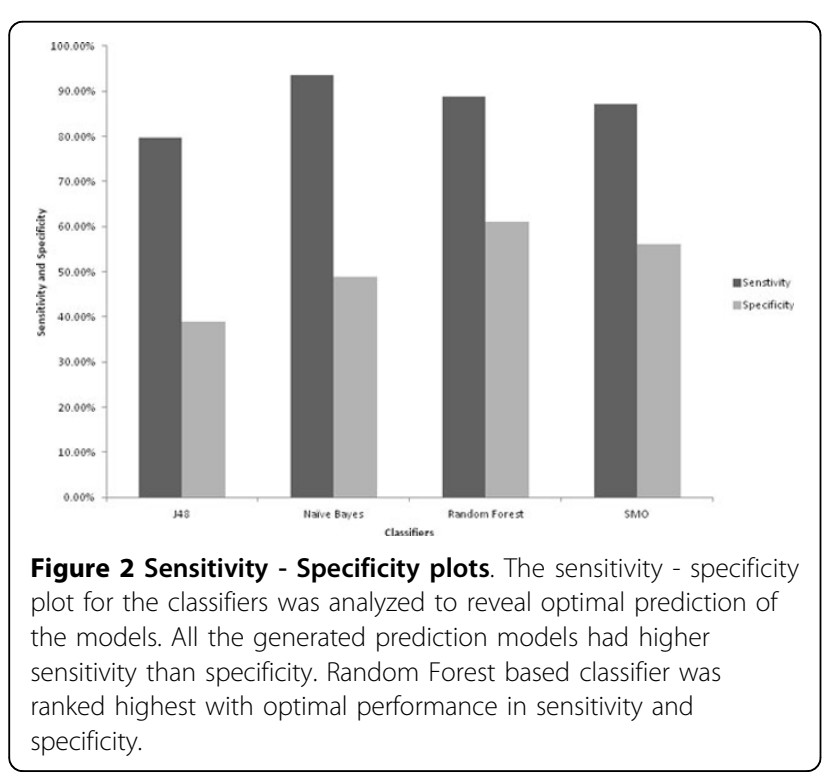

Table 4 Literature validation of the genes selected by feature selection

\begin{tabular}{|c|c|}
\hline Category & Genes \\
\hline Renal Cancer & MAPK7, FGFR3, OASL, GUCY2D, GHRH \\
\hline Other Renal Disorder & NOZ2, APOL1 \\
\hline $\begin{array}{l}\text { Cancer Progression in other } \\
\text { cancers }\end{array}$ & $\begin{array}{c}\text { IRF7, FOXA1, GREB1L, TOB1, RTP3, } \\
\text { IER2, RORL }\end{array}$ \\
\hline Biomarker in other cancers & $\begin{array}{l}\text { SHOX, HDGFL1, HUS1B, GNG7, } \\
\text { AP1M1 }\end{array}$ \\
\hline
\end{tabular}

\# Detailed information about the genes selected by feature selection can be found in Additional File 4

The TCGA gene expression data for ccRCC patients has diverse representation of age, gender and tumor stage (Additional File 1). The patients with localized tumors (stage I and II) were class labeled as early stage whereas patients with locally advanced disease (stage III) and metastatic disease (stage IV) as late stage. The TCGA gene expression profile data available for 475 tumor samples and 20,534 genes had no significant batch effects [14] and came with the "curse of dimensionality". An efficient fast filter feature selection method based on predominant correlation (fast correlation based feature selection) largely reduced the feature space from 20,534 genes to 62 gene expression profiles.

To analyze the details of selected training set genes used in development of prediction models for ccRCC, we first performed GO annotations in which the corresponding biological processes displayed very broad but consistency with cancer hallmarks [33]. Secondly, the oncoprint visualization of the selected subset genes displayed alterations in all the genomic profiles in $83.6 \%$ of the TCGA ccRCC cases. Thirdly, ccRCC cases with alterations in the selected genes had better overall

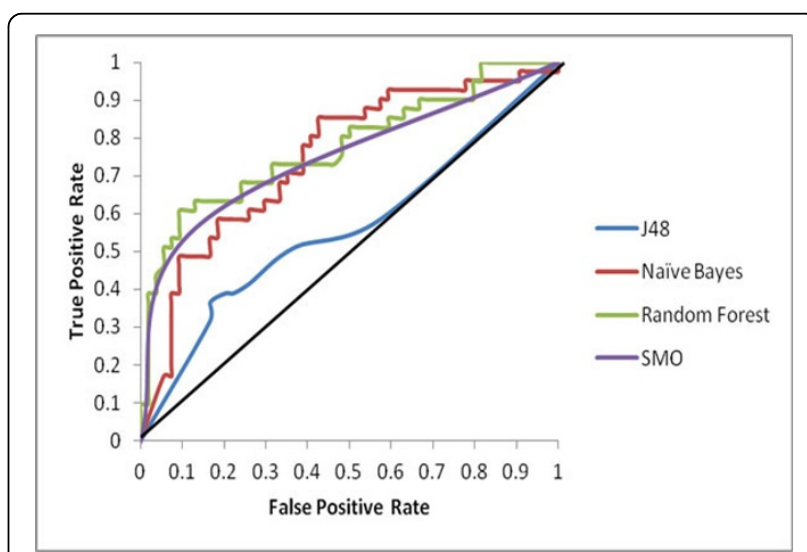

Figure 3 ROC plot. Receivers Operating Characteristic curve (ROC) plot all the four classifiers. Amongst all the prediction models, Random Forest achieved the maximum value for area under Receivers Operating Characteristic curve (auROC) closely followed by Naïve Bayes and SMO. $J 48$ had the least auROC. Scalar values of auROC are given in Table 3. 
survival than cases without alteration in these genes. Lastly, the literature mining of the selected genes revealed that 42 out of 62 genes are already reported in literature for its involvement in cancer progression or detection, either ccRCC or any other type of cancer. This indeed increased our confidence on the applicability of 62 selected genes for development of prediction model as well as the prioritized list to be analyzed for therapeutic and prognostic potential in case of ccRCC.

We found that the prediction model based on Random Forest algorithm is the best classifier followed by Naive Bayes and SMO, which performed reasonably better than J48 based prediction model. The results demonstrate that early stage and late stage ccRCC tumors can be classified with high sensitivity using gene expression profiles. Our observations re-emphasizes the fact that machine learning based models will play important role in the developing field of predictive and personalized medicine.

Our study also illustrates a new method for classification in ccRCC, wherein tumor stage information can be derived from the molecular features, instead of tumor size. In future, availability of additional ccRCC tumor patients and inclusion of more feature vectors like miRNA expressions, protein expression and SNP profiling data could further enhance the accuracy of the prediction models given the fact that ccRCC tumors typically have heterogeneous molecular profiles. Though we tested the prediction models on independent testing datasets of 95 patients, the outcome of such models needs to be interpreted judiciously before incorporation into a clinical set up. We anticipate that the selected gene expression features and the prediction models developed in this study would expedite the challenge for discovering molecular prognosis factors and stage progression molecular signatures in ccRCC tumors.

\section{Conclusion}

An empirical approach has been employed in this study to develop classification model for tumor stage progression in ccRCC based on gene expression profiles. We have identified a subset of 62 genes by feature selection, the expression profiles of which predominantly correlates with tumor-stage of the patient given the heterogeneous data in terms of tumor stage, age group and gender of the patient. We report that Random Forest based prediction model accurately and reliably classifies patient tumor stages. We anticipate that such prediction models based on the molecular correlates could contribute to the optimal management of patients in ccRCC. Currently, the prediction models generated are available upon request to the authors. To the best of our knowledge, we are reporting the first prediction model to classify ccRCC tumor stage based on gene expression profiles.

\section{Additional material}

Additional file 1: Data characteristics of ccRCC level 3 information for TCGA patients for KIRC. This file consists of 2 figures - Gender wise stage distribution of the TCGA patients; and Age distribution of the patients. The figures are in a Portable Document Format (PDF) and can be viewed with any standard PDF viewer.

Additional file 2: Training dataset. This file consists of Training dataset used in this study. The file is in a Comma Separated Value (CSV) format with 20,534 gene's expression in RPKM in column and 380 patients as rows. The file is in CSV format.

Additional file 3: Testing dataset. This file consists of Testing dataset used in this study. The file is in a Comma Separated Value (CSV) format with 20,534 gene's expression in RPKM in column and 95 patients as rows. The file is in CSV format.

Additional file 4: Gene symbols and gene names and literature validation of the selected genes after feature selection. This file consists of 62 genes selected by feature selection in the study and gene names annotated by DAVID-gene name batch viewer (http://david.abcc. ncifcrf.gov/). Literature validation of 42 out of 62 genes for involvement in renal cancer, renal disease, including disease association for cancer progression, \& biomarkers in other cancers is given from literature and Gene Cards (http://www.genecards.org/). The file is in .docx format and can be viewed using any document viewer like Microsoft Word.

Additional file 5: Distribution plot of the expression values selected 62 genes. This file consists of expression value distribution plots generated by Weka Explorer of final 62 genes for the class label "Early Stage" and "Late Stage". The file is in .docx format and can be viewed using any document viewer like Microsoft Word.

Additional file 6: Analysis of the 62 selected genes for GO annotations - Biological Process; Oncoprint and Overall Survival Kaplan-Meier Estimate from cBioportal for Cancer Genomics. This file consists of 3 figures - Bar chart of GO biological process categories of the selected 62 genes; Screen Shot of Oncoprint for genomic alterations in selected 62 genes in ccRCC cases in cBioPortal for Cancer Genomics age distribution of the patients; and Survival Kaplan-Meier Estimate for selected 62 genes in ccRCC cases in cBioPortal for Cancer Genomics. The figures are in a Portable Document Format (PDF) and can be viewed with any standard PDF viewer.

\section{Competing interests}

The authors declare that they have no competing interests.

\section{Authors' contributions}

ZJ and DG designed the study. ZJ implemented the study. ZJ and DG have prepared the manuscript and approve the manuscript.

\section{Acknowledgements}

The authors thank Department of Biotechnology (DBT, India) grant (BT/BI/25/ 001/2006) for "Bioinformatics Infrastructure Facility" at ICGEB, New Delhi. ZJ acknowledges University Grants Commission (UGC, India) for Senior Research Fellowship.

This article has been published as part of BMC Proceedings Volume 8 Supplement 6, 2014: Proceedings of the Great Lakes Bioinformatics Conference 2014. The full contents of the supplement are available online at http://www.biomedcentral.com/bmcproc/supplements/8/S6.

\section{Declarations}

Publication of this work was funded by International Centre for Genetic Engineering and Biotechnology, New Delhi, India.

Published: 13 October 2014

\section{References}

1. Graves A, Hessamodini H, Wong G, Lim W: Metastatic renal cell carcinoma: update on epidemiology, genetics, and therapeutic modalities. 2013. 
2. Jayson $\mathrm{M}$, Sanders $\mathrm{H}$ : Increased incidence of serendipitously discovered renal cell carcinoma. Urology 1998, 51:203-205.

3. Ng CS, Wood CG, Silverman PM, Tannir NM, Tamboli P, Sandler CM: Renal Cell Carcinoma: Diagnosis, Staging, and Surveillance. American Journal of Roentgenology 2008, 191:1220-1232.

4. Cohen HT, McGovern FJ: Renal-cell carcinoma. New England Journal of Medicine 2005, 353:2477-2490.

5. Seliger B, Dressler SP, Lichtenfels R, Kellner R: Candidate biomarkers in renal cell carcinoma. Proteomics 2007, 7:4601-4612.

6. Hudson TJ, Anderson W, Aretz A, Barker AD, Bell C, Bernabé RR, Bhan M, Calvo F, Eerola I, Gerhard DS: International network of cancer genome projects. Nature 2010, 464:993-998.

7. Chin L, Andersen JN, Futreal PA: Cancer genomics: from discovery science to personalized medicine. Nature medicine 2011, 17:297-303.

8. Chin L, Hahn WC, Getz G, Meyerson M: Making sense of cancer genomic data. Genes \& development 2011, 25:534-555.

9. Cruz JA, Wishart DS: Applications of machine learning in cancer prediction and prognosis. Cancer informatics 2006, 2:59.

10. McCarthy JF, Marx KA, Hoffman PE, Gee AG, O'Neil P, Ujwal ML, Hotchkiss J: Applications of Machine Learning and High-Dimensional Visualization in Cancer Detection, Diagnosis, and Management. Annals of the New York Academy of Sciences 2004, 1020(1):239-262.

11. Lam JS, Shvarts O, Leppert JT, Figlin RA, Belldegrun AS: Renal cell carcinoma 2005: new frontiers in staging, prognostication and targeted molecular therapy. The Journal of urology 2005, 173:1853-1862.

12. Tsui K-H, Shvarts O, Smith RB, Figlin RA, deKernion J, Belldegrun A: Prognostic indicators for renal cell carcinoma: a multivariate analysis of 643 patients using the revised 1997 TNM staging criteria. The Journal of urology 2000, 163:1090-1095.

13. Levy DA, Slaton JW, Swanson DA, Dinney CP: Stage specific guidelines for surveillance after radical nephrectomy for local renal cell carcinoma. The Journal of urology 1998, 159:1163-1167.

14. Network CGAR: Comprehensive molecular characterization of clear cell renal cell carcinoma. Nature 2013, 499:43-49.

15. Hall M, Frank E, Holmes G, Pfahringer B, Reutemann P, Witten IH: The WEKA data mining software: an update. ACM SIGKDD Explorations Newsletter 2009, 11:10-18.

16. Yu L, Liu H: Feature selection for high-dimensional data: A fast correlation-based filter solution. ICML 2003, 856-863.

17. Witten IH, Frank E: Data Mining: Practical machine learning tools and techniques Morgan Kaufmann; 2005.

18. Wang J, Duncan D, Shi Z, Zhang B: WEB-based GEne SeT AnaLysis Toolkit (WebGestalt): update 2013. Nucleic acids research 2013.

19. Cerami E, Gao J, Dogrusoz U, Gross BE, Sumer SO, Aksoy BA, Jacobsen A, Byrne CJ, Heuer ML, Larsson E: The cBio cancer genomics portal: an open platform for exploring multidimensional cancer genomics data. Cancer discovery 2012: :401-404.

20. Gao J, Aksoy BA, Dogrusoz U, Dresdner G, Gross B, Sumer SO, Sun Y, Jacobsen $A$, Sinha R, Larsson E: Integrative analysis of complex cancer genomics and clinical profiles using the cBioPortal. Science signaling 2013, 6:pl1.

21. Quinlan JR: C4.5: programs for machine learning Morgan Kaufmann Publishers Inc; 1993.

22. Friedman N, Geiger D, Goldszmidt M: Bayesian network classifiers. Machine learning 1997, 29:131-163.

23. Keerthi SS, Shevade SK, Bhattacharyya C, Murthy KRK: Improvements to Platt's SMO algorithm for SVM classifier design. Neural Computation 2001, 13:637-649.

24. Platt JC: 12 Fast Training of Support Vector Machines using Sequential Minimal Optimization. 1999.

25. Breiman L: Random Forests. Machine learning 2001, 45:5-32.

26. Chou KC: Some remarks on protein attribute prediction and pseudo amino acid composition. Journal of theoretical biology 2011, 273:236-247.

27. Sokolova M, Lapalme G: A systematic analysis of performance measures for classification tasks. Information Processing \& Management 2009, 45:427-437.

28. Sun Y, Kamel MS, Wong AKC, Wang Y: Cost-sensitive boosting for classification of imbalanced data. Pattern Recognition 2007, 40:3358-3378.

29. Takahashi M, Rhodes DR, Furge KA, Kanayama Ho, Kagawa S, Haab BB, Teh BT: Gene expression profiling of clear cell renal cell carcinoma: gene identification and prognostic classification. Proceedings of the National Academy of Sciences 2001, 98:9754-9759.
30. Zhao H, Ljungberg B, Grankvist K, Rasmuson T, Tibshirani R, Brooks JD: Gene expression profiling predicts survival in conventional renal cell carcinoma. PLoS medicine 2005, 3:e13.

31. Brannon AR, Reddy A, Seiler M, Arreola A, Moore DT, Pruthi RS, Wallen EM, Nielsen ME, Liu H, Nathanson KL: Molecular stratification of clear cell renal cell carcinoma by consensus clustering reveals distinct subtypes and survival patterns. Genes \& cancer 2010, 1:152-163.

32. Skubitz KM, Zimmerman W, Kammerer R, Pambuccian S, Skubitz AP: Differential gene expression identifies subgroups of renal cell carcinoma. Journal of Laboratory and Clinical Medicine 2006, 147:250-267.

33. Hanahan D, Weinberg RA: Hallmarks of cancer: the next generation. Cell 2011, 144:646-674

doi:10.1186/1753-6561-8-S6-S2

Cite this article as: Jagga and Gupta: Classification models for clear cell renal carcinoma stage progression, based on tumor RNAseq expression trained supervised machine learning algorithms. BMC Proceedings 2014 8(Suppl 6):S2.

\section{Submit your next manuscript to BioMed Central and take full advantage of:}

- Convenient online submission

- Thorough peer review

- No space constraints or color figure charges

- Immediate publication on acceptance

- Inclusion in PubMed, CAS, Scopus and Google Scholar

- Research which is freely available for redistribution
Ciomed Central 